\title{
Internações por diabetes mellitus no estado do Pará: distribuição espacial e fatores associados ao óbito
}

RESUMO | Objetivo: analisar a taxa de internação por Diabetes mellitus no Pará. Método: Estudo analítico, de abordagem quantitativo. Os dados das internações hospitalares do período de 2008 a 2017 foram extraídos do Sistema de Informação Hospitalar do Sistema Único de Saúde, por meio do programa Tabwin. Para análise foram utilizados os programas Bioestat, Tabwin e Excel. Resultados: Foram registradas 53.954 internações e 1.950 óbitos ocorridos durante a internação pela doença. O maior risco de óbito na internação pela doença foi associado aos homens, pessoas com idade acima de 70 anos, e nas complicações agudas e de longo prazo. A taxa de internação ao longo de todo o período foi crescente nas Regiões de Saúde do Marajó I, II e Araguaia. Conclusão: Houve um crescimento das taxas de internação e mortalidade durante a internação por Diabetes Mellitus até o ano de 2015 no Pará e variação entre as regiões de saúde.

Palavras-chaves: Hospitalização; Diabetes Mellitus; Mortalidade.

ABSTRACT | Objective: analyze the hospitalization rate for Diabetes mellitus in Pará. Method: Analytical study, with a quantitative approach. Data from hospital admissions from 2008 to 2017 were extracted from the Hospital Information System of the Unified Health System, through the Tabwin program. For the analysis, the programs Bioestat, Tabwin and Excel were used. Results: There were 53,954 hospitalizations and 1,950 deaths during hospitalization. The highest risk of death in hospitalization for the disease was associated with men, people over 70 years of age, and in acute and long-term complications. The hospitalization rate throughout the period was increasing in the Health Regions of Marajó I, II and Araguaia. Conclusion: There was an increase in hospitalization rates and mortality during hospitalization for Diabetes mellitus up to 2015 in Pará and variation among health regions.

Keywords: Hospitalization; Diabetes Mellitus; Mortality.

RESUMEN | Objetivo: analizar la tasa de internación por Diabetes mellitus en Pará. Método: Estudio analítico, de abordaje cuantitativo. Los datos de las internaciones hospitalarias del período de 2008 a 2017 fueron extraídos del Sistema de Información Hospitalaria del Sistema Único de Salud, a través del programa Tabwin. Para el análisis se utilizaron los programas Bioestat, Tabwin y Excel. Resultados: Se registraron 53.954 internaciones y 1.950 muertes ocurridas durante la internación por la enfermedad. El mayor riesgo de muerte en la internación por la enfermedad fue asociado a los hombres, personas mayores de 70 años, y en las complicaciones agudas ya largo plazo. La tasa de internación a lo largo de todo el período fue creciente en las Regiones de Salud del Marajó I, II y Araguaia. Conclusión: Hubo un crecimiento de las tasas de internación y mortalidad durante la internación por Diabetes Mellitus hasta el año 2015 en Pará y variación entre las regiones de salud.

Descriptores: Hospitalización; Diabetes Mellitus; Mortalidad.

\section{Cléo da Costa Araújo}

Residente do Programa de residência multiprofissional de Saúde da Família da Universidade Estadual do Pará. Graduação em Enfermagem.

\section{Carlos Leonardo Figueiredo Cunha}

Professor Adjunto da Universidade Federal do Pará. Enfermeiro. Doutor em Saúde Coletiva.

\section{Rubenilson Caldas Valois}

Professor Adjunto da Universidade do Estado do Pará. Enfermeiro. Doutor em Doenças Tropicais.

\section{Eliã Pinheiro Botelho}

Professor Adjunto da Universidade Federal do Pará. Enfermeiro. Doutorado em Ciências Biológicas.

\section{Jéssica Soares Barbosa}

Discente do Programa de Iniciação Científica da Universidade Federal do Pará. Acadêmica de Enfermagem.

\section{Glenda Roberta Oliveira Naiff Ferreira}

Professora Adjunto da Universidade Federal do Pará. Enfermeira. Doutora em Biologia dos Agentes Infecciosos e Parasitários.

Recebido em: 30/05/2019

Aprovado em: 30/05/2019
INTRODUÇÃO

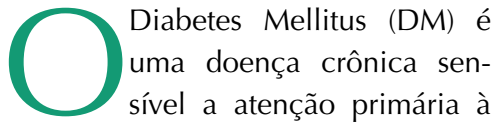
saúde relacionada ao maior risco de internação e tempo de permanência hospitalar, representando um impacto econômico expressivo nos gastos de recursos da saúde pública e na qualidade de vida das pessoas ${ }^{(1-4)}$.

As complicações agudas decorrentes das alterações no nível glicêmico e aquelas a longo prazo, como alterações cardiovasculares, neurológicas, renais e as amputações, contribuem para o aumento das internações hospitalares por DM. Ademais, esse perfil de morbidade, algumas características individuais e a falta de cuidado e autocuidado contribuem para o agravamento da condição e 
aumento do risco de óbito na internação por $\mathrm{DM}^{(3-5)}$.

Apesar das características epidemiológicas das internações por DM variarem entre diferentes grupos populacionais, estudos $^{(2,3,5)}$ demonstram que a frequência de internação é maior nas pessoas com DM que possuem idade acima de 60 anos, baixa renda salarial e escolaridade. Quanto ao sexo, verificam-se diferenças, como demonstrado em estudos realizados na China e no Canadá em que foi encontrada maior frequência nos homens, enquanto que nos Estados Unidos, nas mulheres ${ }^{(2,6-7)}$. Essas diferenças também foram encontradas em estudos realizados no Brasil ${ }^{(5,8-9)}$.

Torna-se claro que a magnitude das internações hospitalares e da mortalidade por DM estão relacionadas as especificidades de cada território. Isso pode decorrer por questões associadas ao acesso à rede de serviço de saúde e o impacto das respostas sociais produzidas pelo sistema de saúde. Entre distritos sanitários de um mesmo município, a taxa de internação varia de 26,6 a 83,8 internações por dez mil habitantes ${ }^{(1,10-12)}$. Esses dados reforçam a importância desses indicadores como importantes instrumentos na avaliação da atenção primária e da utilização da rede de serviço da atenção terciária ${ }^{(1)}$.

Ao longo dos anos, o Estado do Pará seguiu a tendência observada no Brasil de expansão da Estratégia Saúde da Família (ESF) e redução das Internações por Condições Sensíveis à Atenção Primária (ICSAP), da qual faz parte as internações por $\mathrm{DM}^{(1,13)}$. No entanto, a cobertura de Atenção Primária à Saúde (APS) não atende às necessidades da população quanto ao acesso prioritário a rede de serviço, além de existir um desequilíbrio na distribuição da oferta de serviços de saúde da rede pública, principalmente, para áreas mais remotas, promovendo desigualdades no acesso a assistência à saúde que podem refletir nos indicadores de saúde ${ }^{(13-14)}$.

Mesmo mediante tais fatos, não foram encontrados estudos sobre a taxa de internação por DM associados com ta- xas de óbitos no Estado do Pará e padrão distribuição das internações por região de saúde. A variação de resultados encontrados em estudos prévios realizados em diferentes lugares salienta a necessidade de se estudar os indicadores localmente, sendo que indagamos qual a magnitude e padrão de distribuição das internações por DM no Pará e suas regiões de saúde? A partir da questão norteadora, nesse estudo propusemos analisar a taxa de internação por DM no Estado do Pará, assim como por regiões de saúde, no período de 2008 a 2017, e associar o óbito na internação e as características epidemiológicas das pessoas que internaram por DM.

\section{METODOLOGIA}

Trata-se de um estudo analítico, de abordagem quantitativa, transversal sobre as internações por DM ocorridas no Estado do Pará, no período de 2008 a 2017. O Pará, localizado na Região Norte do Brasil, tem área de 1.247.955, 238 km2 e uma população de 7.581 .051 pessoas, conforme o censo de 2010, distribuídas em 144 municípios, cada um vinculado a uma das treze regiões de saúde $\mathrm{e}^{(13-14)}$.
Os dados foram coletados por meio de download disponibilizado pelo Departamento de Informática do SUS (Datasus), cujos dados foram extraídos entre setembro e outubro de 2018, utilizando o programa Tabwin 4.1.5. O Sistema de Informações Hospitalares do Sistema Único de Saúde (SIH/SUS) foi a fonte de dados sobre as internações hospitalares por Diabetes Mellitus. A fonte de dados populacionais e os arquivos de mapa foram extraídos do Instituto Brasileiro de Geografia e Estatística (IBGE).

Foram selecionadas apenas as internações por DM, que incluíram o DM com coma ou cetoacidose (E100-E101,E110-E111,E120-E121,E130-E131,E140-E141), com complicações-renais, oftalmológicas, etc (E102-E108,E112-E118,E122-E128,E132-E138,E142-E148) e sem complicações (E109,E119,E129,E139,E149). Para extração das variáveis, foi adotado o ano de internação, o sexo, a faixa etária, cor/ raça, evolução clínica, região de saúde de residência e óbitos na internação. Os dados foram armazenados em planilha

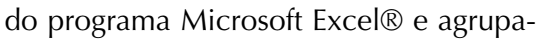
dos em biênios para reduzir as flutuações ocorridas ano a ano(15).

\section{Figura 1. Regiões de saúde do Pará. Belém, PA, Brasil, 2018.}

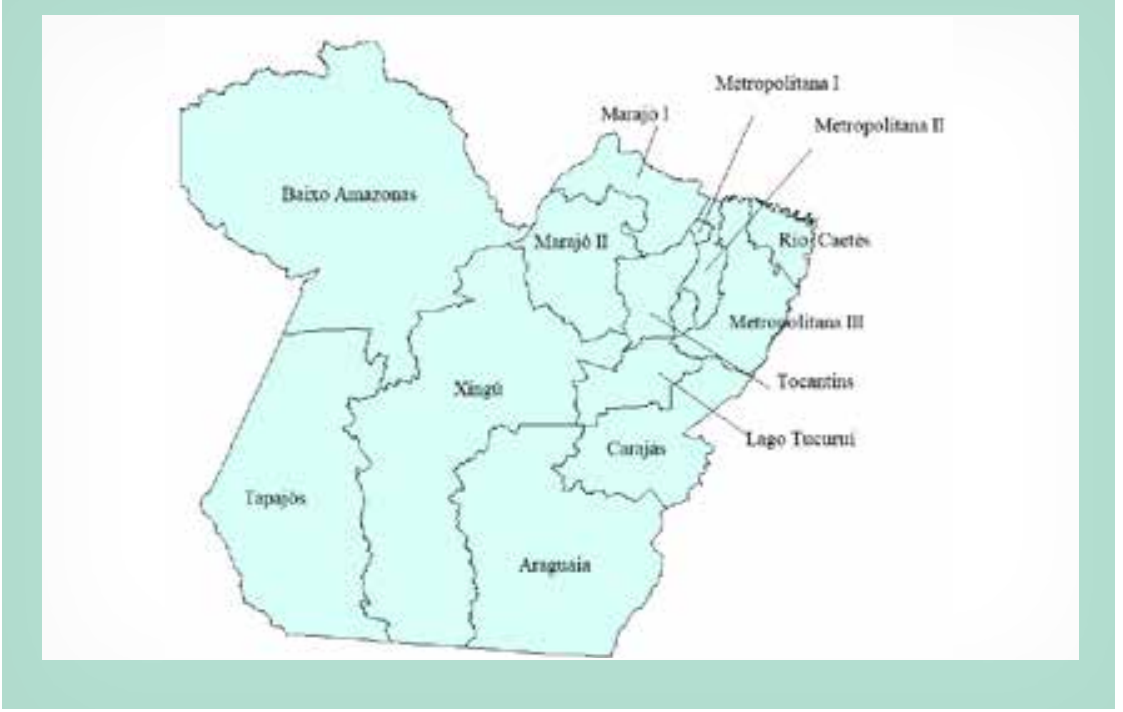

Fonte: Tabwin IBGE. 
O cálculo da taxa bruta de internação seguiu o seguinte método de cálculo: número de internações dividido pela população residente, multiplicado por dez mil habitantes (hab). Enquanto a taxa bruta de mortalidade foi calculada por 100 mil hab. A variação percentual da frequência absoluta e das taxas de internação foram calculadas seguindo o método de cálculo descrito em estudo prévio ${ }^{(16)}$ : valor registrado no último ano menos o valor registrado no primeiro ano, em seguida dividiu-se o resultado pelo valor registrado no primeiro ano e, por último, multiplicou-se o resultado por 100 .

Para análise da distribuição espacial, foi realizada padronização das taxas pelo método direto para retirar a influência das diferenças devidas das faixas etárias, sendo a população do estado do Pará do ano de 2010 adotada como padrão, conforme realizado em estudos prévios ${ }^{(11,17)}$. Para conhecer os padrões de distribuição geográfica das internações pela doença estudada, os dados da taxa padronizada de internação de cada região de saúde, previamente calculados, foram transcritos para uma tabela do Tabwin. Posteriormente, foram elaborados mapas temáticos, do tipo coroplético.

Para associação entre o perfil epidemiológico das internações por DM e o óbito na internação foi realizado Odds Ratio. As análises estatísticas foram realizadas no programa Bioestat versão 5.3, programa de acesso irrestrito. Foi adotado o nível de significância estatística associada $p<0,05$. Os gráficos e tabelas foram realizados no programa Microsoft Excel ${ }^{\circledR}$. Os mapas foram elaborados no programa Tabwin 4.1.5.

Os pesquisadores não tiveram contato com as pessoas que internaram, uma vez que foram utilizadas fontes de dados secundários de domínio público, o que dispensa da apreciação por Comitê de Ética em Pesquisa em conformidade com o parágrafo único do Artigo $1^{\circ}$ da Resolução n. ${ }^{\circ}$ 510, de 07 de abril de 2016, do Conselho Nacional de Saúde (CNS). Os pesquisadores respeitaram todas as diretrizes éticas de pesquisa com seres humanos, conforme recomenda a Resolução do CNS n. ${ }^{\circ}$ 466, de 12 de dezembro de 2012.

\section{RESULTADOS}

Entre 2008 a 2017, foram registradas 53.954 internações por DM no Pará. Considerando todo o período, a taxa de internação foi de 71,2 internações por 10 mil habitantes, com aumento percentual de $22,2 \%$ internações entre 2008 a 2017. Ao analisarmos as taxas de internações nos biênios, verifica-se que houve aumento progressivo até 2014-2015, período que foi registrado a maior taxa da série temporal (16,1/10.000 hab.). No biênio subsequente, 2016-2017, houve redução de $8,1 \%$ nas internações registradas (Tabela 1).

Em relação aos óbitos, foram registrados 1950 óbitos cuja causa de internação foi por DM, nesse período a taxa de mortalidade na internação foi de 25,7 óbitos (/100 mil hab.). A variação percentual dos óbitos entre 2008 a 2017 registrou aumento de 20,8\%. Verifica-se que a trajetória da taxa de mortalidade durante os biênios é similar ao observado na taxa de internação, com aumento até o período de 2014 a 2015, seguido de redução no período seguinte.

Tabela1 - Distribuição por biênios das Taxas bruta de internação por 10 mil e mortalidade por 100 mil habitantes por Diabetes Mellitus. Belém, PA, Brasil, 2008-2017.

\begin{tabular}{|c|c|c|c|c|c|c|c|c|c|c|}
\hline Taxa Bruta & \multicolumn{2}{|c|}{ 2008-2009 } & \multicolumn{2}{|c|}{ 2010-2011 } & \multicolumn{2}{|c|}{$2012-2013$} & \multicolumn{2}{|c|}{$2014-2015$} & \multicolumn{2}{|c|}{ 2016-2017 } \\
\hline Internação* & 9176 & 12,1 & 10095 & 13,3 & 11260 & 14,9 & 12207 & 16,1 & 11216 & 14,8 \\
\hline Mortalidade** & 322 & 4,2 & 364 & 4,8 & 430 & 5,7 & 445 & 5,9 & 389 & 5,1 \\
\hline
\end{tabular}

Nota: *Taxa bruta de internação por 10 mil. ** Taxa bruta de mortalidade por 100 mil. Fonte: SIH/SUS

A Tabela 2 apresenta a variação percentual e distribuição das internações DM, segundo características epidemiológicas agrupadas por biênios. Entre 2008 a 2017, o maior número de internações foi registrado no sexo feminino com 56,8\% (30.623), sendo predominante em todos os biênios. No entanto, as internações do sexo masculino apresentaram aumento percentual de $32,1 \%$, sendo supe- rior ao sexo feminino.

Considerando as internações por DM, segundo a faixa etária no período estudado, observa-se maior número de internações na faixa etária de 60 a 69 anos com 14.279 e predominou em todos os biênios. A trajetória linear das internações entre 2008 a 2017 demonstra que os extremos de idade (menos de 1 ano a 9 anos e idade igual ou superior a 80 anos) apresen- taram aumento progressivo no número de internações.

O resultado da variação percentual, entre 2008 e 2017, demonstra que o maior aumento no percentual de internação ocorreu na faixa etária igual ou superior a 80 anos. A faixa etária de 40 a 49 anos registrou a maior redução na variação percentual. Ao analisarmos a dinâmica das internações em cada biênio, verifica-se que o aumento do número de 
internações seguiu, conforme a elevação da idade até a faixa etária de 60 a 69 anos, a partir dessa faixa etária foi observada redução no número de internações.

Em todo o período estudado, há uma maior frequência de internação entre as pessoas declaradas pardas. A menor fre- quência foi entre os indígenas e amarelos. Contudo, ao analisarmos a variação percentual, as internações entre os declarados amarelos tiveram aumento percentual. Constata-se que o maior percentual de redução foi entre os indígenas.

Considerando a evolução clínica, houve maior número de internações de complicações a longo prazo. A maior variação percentual ocorreu entre as internações sem complicações com aumento e registra-se redução percentual nas internações associadas a complicações agudas.

Tabela 2 - Distribuição das internações por Diabetes Mellitus por biênios, segundo características epidemiológicas. Belém, PA, Brasil, 2008-2017.

\begin{tabular}{lccccccccccc}
$\begin{array}{c}\text { Características epide- } \\
\text { miológicas }\end{array}$ & $\mathbf{2 0 0 8 - 2 0 0 9}$ & 2010-2011 & 2012-2013 & 2014-2015 & 2016-2017 & Variação \\
\multicolumn{1}{c}{ percentual }
\end{tabular}

Faixa etária

\begin{tabular}{|c|c|c|c|c|c|c|c|c|c|c|c|}
\hline Menos 1-9 & 102 & 1,1 & 100 & 1,0 & 91 & 0,8 & 101 & 0,8 & 116 & 1,0 & 13,7 \\
\hline $10-19$ & 162 & 1,8 & 157 & 1,6 & 202 & 1,8 & 231 & 1,9 & 187 & 1,7 & 15,4 \\
\hline $20-29$ & 256 & 2,8 & 280 & 2,8 & 262 & 2,3 & 282 & 2,3 & 243 & 2,2 & $-5,1$ \\
\hline $40-49$ & 1289 & 14,0 & 1302 & 12,9 & 1363 & 12,1 & 1425 & 11,7 & 1203 & 10,7 & $-6,7$ \\
\hline $50-59$ & 2143 & 23,4 & 2189 & 21,7 & 2512 & 22,3 & 2773 & 22,7 & 2504 & 22,3 & 16,8 \\
\hline $70-79$ & 1719 & 18,7 & 1924 & 19,1 & 2200 & 19,5 & 2490 & 20,4 & 2307 & 20,6 & 34,2 \\
\hline Acima de 80 & 627 & 6,8 & 916 & 9,1 & 1019 & 9,0 & 1049 & 8,6 & 1096 & 9,8 & 74,8 \\
\hline
\end{tabular}

Cor/Raça

\begin{tabular}{lcccccccccccc} 
Branca & 406 & 8,0 & 305 & 6,0 & 271 & 4,7 & 242 & 3,2 & 225 & 3,3 & $-44,6$ \\
\hline Negros & 218 & 4,3 & 175 & 3,4 & 141 & 2,5 & 145 & 1,9 & 130 & 1,9 & $-40,4$ & 92 \\
Parda & 4309 & 84,5 & 4610 & 90,4 & 5312 & 92,5 & 7053 & 93,7 & 6214 & 92,0 & 44,2 \\
\hline Amarela & 18 & 0,4 & 9 & 0,2 & 8 & 0,1 & 77 & 1,0 & 175 & 2,6 & 872,2 \\
Indígena & 146 & 2,9 & 3 & 0,1 & 9 & 0,2 & 9 & 0,1 & 11 & 0,2 & $-92,5$ \\
\hline NI $^{*}$ & 4079 & & 4993 & & 5519 & & 4681 & & 4461 &
\end{tabular}

Evolução clínica

\begin{tabular}{lcccccccccccc} 
Outras complicações & 6020 & 65,6 & 6149 & 60,9 & 6725 & 59,7 & 7637 & 62,6 & 7060 & 62,9 & 17,3 & 5,3 \\
\hline Coma ou cetoacidose & 914 & 10,0 & 666 & 6,6 & 685 & 6,1 & 733 & 6,0 & 629 & 5,6 & $-31,2$ \\
\hline Sem complicações & 2242 & 24,4 & 3280 & 32,5 & 3850 & 34,2 & 3837 & 31,4 & 3527 & 31,4 & 57,3
\end{tabular}

Nota: *Não Informado: Não considerado para cálculo da frequência relativa variação percentual.

Fonte: SIH/SUS

A associação das características epidemiológicas e o óbito durante a internação por DM no Pará entre 20082017 demonstrou que, apesar do sexo feminino ter o maior número de inter- nações, a probabilidade de óbito entre os homens é superior as mulheres. Quanto à faixa etária, só foi observada maior chance de óbitos nas faixas etárias de 20 a 29 anos, 70 a 79 anos e nas pessoas acima de 80 anos. Em relação as internações por complicações, o risco de óbito foi por complicações de longo prazo e nas internações por coma ou cetoacidose. Não houve as- 
sociação estatisticamente significante entre óbitos nas internações por DM com cor/raça.

Considerando as diferenças regionais existentes no Pará. A Figura 2 apresenta a magnitude e padrão de distribuição espacial das internações por DM, sendo apresentados os dados da taxa padronizada. A trajetória temporal linear referente ao período entre 2008 a 2017 das internações demonstrou que houve aumento progressivo nas taxas de internação (/10 mil habitantes) nas pessoas precedentes das regiões do Marajó I $(2,6 ; 3,2 ; 3,3 ; 8,5$;
11,9), Marajó II (3,9; 6,1; 11,5; 12,6; 14,) e Araguaia $(16,9 ; 21,7 ; 23,7 ; 26,9 ; 29,1)$. Nas regiões de saúde do Xingú, Lago Tucuruí, metropolitana II e Tapajós houve aumento até o biênio de 2014 a 2015.

Considerando a variação percentual em números absolutos, os maiores aumentos nas internações foram de procedentes da Marajó I com 345,3\% e Marajó II com 275\%, no período de 2008 a 2017. Redução na variação percentual foi observado na região de saúde Metropolitana II, com $6,3 \%$ de redução nas internações; Metropolitana II (-5,5\%) e Rio
Caetés (-0,3\%). Dados não apresentados em tabelas ou figuras.

\section{DISCUSSÃO}

Nossos achados demonstram aumento da taxa de internação e mortalidade durante a internação no Pará, fornecendo evidências sobre os fatores associados ao óbito na internação.

No Paraná, entre 2000 a 2012, foram registrados 111.402 internações decorrentes do DM, sendo duas vezes maior que o registrado no estudo ${ }^{(18)}$.

Tabela 3 - Associação entre o perfil epidemiológico e os óbitos entre as pessoas que internaram por Diabetes Mellitus. Belém, PA, Brasil, 2008-2017.

\section{Características epidemiológicas}

\section{Sexo}

$\begin{array}{cccccc}\text { Feminino } & 29560 & 1063 & \text { Ref. } & \\ \text { Masculino } & 22444 & 887 & 1.09 & 1,00-1,20 & 0,0439\end{array}$

\section{Faixa etária}

\begin{tabular}{|c|c|c|c|c|c|c|}
\hline & $0-9$ & 501 & 9 & Ref. & & \\
\hline & $10-19$ & 919 & 20 & 1,21 & $0,54-2,68$ & 0,7812 \\
\hline & $20-29$ & 1274 & 49 & 2,14 & $1,04-4,39$ & 0,0481 \\
\hline & $30-39$ & 2782 & 71 & 1,42 & $0,70-2,86$ & 0,4063 \\
\hline & $40-49$ & 6427 & 155 & 1,34 & $0,68-2,64$ & 0,4830 \\
\hline & $50-59$ & 11825 & 296 & 1,39 & $0,71-2,72$ & 0,4072 \\
\hline & $70-79$ & 10156 & 484 & 2,65 & $1,36-5,16$ & 0,0040 \\
\hline & Acima de 80 anos & 4324 & 383 & 4,93 & $2,52-9,61$ & $<0,0001$ \\
\hline \multicolumn{7}{|l|}{ Raça } \\
\hline & Amarela/Indígena & 452 & 13 & Ref. & & \\
\hline & Negros & 795 & 14 & 0,61 & $0,28-1,31$ & 0,2852 \\
\hline & $\mathrm{NI}^{*}$ & 22704 & 1029 & & & \\
\hline \multicolumn{7}{|l|}{ Evolução Clínica } \\
\hline & Sem complicação & 16377 & 359 & Ref. & & \\
\hline & Outras complicações & 32272 & 1319 & 1,86 & $1,65-2,09$ & $<0,0001$ \\
\hline & Coma ou cetacidose & 3355 & 272 & 3,69 & $3,14-4,34$ & $<0,0001$ \\
\hline
\end{tabular}

Nota: OR: Odds ratio IC: intervalo de confiança. *Não Informado: Não considerado para cálculo da frequência relativa e para estatística. Fonte: SIH/SUS. 

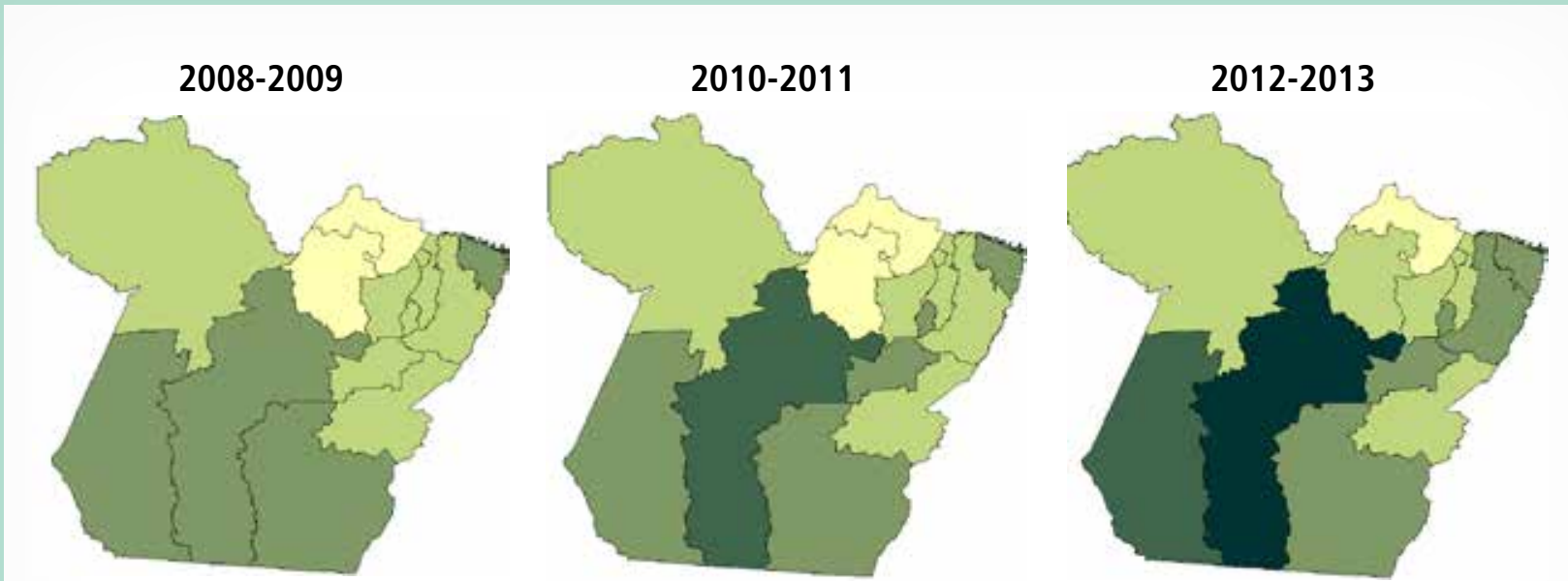

2014-2015

2016-2017
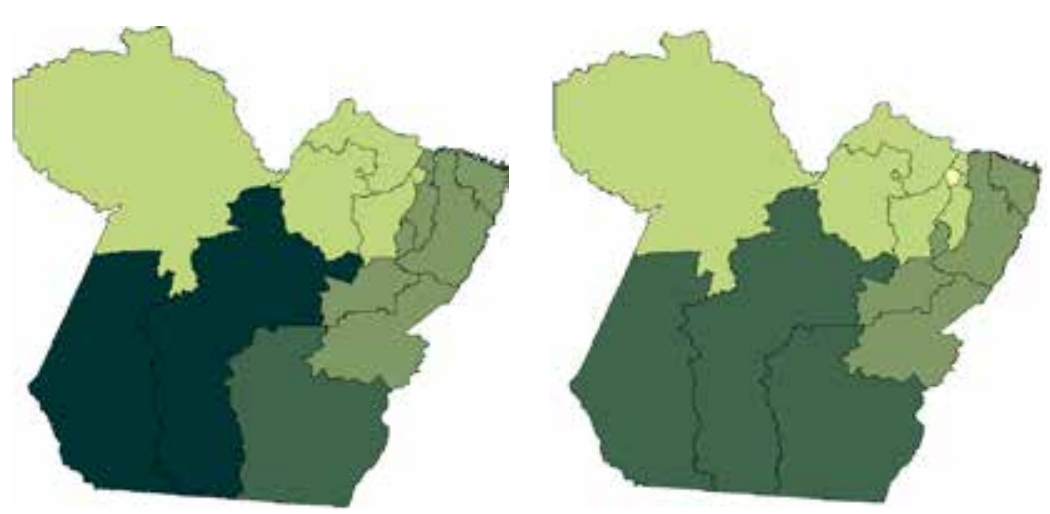

Até 8

8,01 a 16

16,01 a 24

24,01 a 32

32,01 a 40

Fonte: SIH.

Em Rondônia, entre 2012 a 2016, o número de internações foi menor, no entanto, a taxa de internação de 76,6 por 10.000 habitantes foi similar ao nosso estudo ${ }^{(19)}$. Estudos ${ }^{(10,20)}$ realizados na população em geral, em outros países, demonstram que a taxa geral de mortalidade por DM apresentou tendência decrescente. Em sentido contrário, estudo ${ }^{(21)}$ realizado na população em geral em Minas Gerais demonstrou aumento desse indicador. No entanto, esses territórios têm características sociodemográficas, epidemiológicas e de acesso a serviços de saúde diferentes do local do estudo, fatores que devem ser considerados para a análise situacional e planejamento de medidas de prevenção e controle.

O perfil epidemiológico das pessoas que internam por DM varia entre diferentes grupos populacionais. Nossos achados quanto à idade são similares ao encontrado em estudos anteriores realizado Taiwan, Lima, Brasil e Estados Unidos. Contudo, ao contrário do encontrado na população do estudo, em Taiwan os homens foram os mais aco- metidos pela necessidade de internação por $\mathrm{DM}^{(6,22-24)}$.

Assim como no presente estudo, em Lima, no Peru, houve maior frequência de internação entre mulheres. Esse perfil também foi observado no Paraná, porém com tendência crescente na taxa de internação em homens. Nos Estados Unidos, a internação acomete, principalmente, brancos e negros. Outras características das pessoas que internam incluem o baixo nível socioeconômico e estilo de vida, que juntos elevam tempo de internação ${ }^{(6,22-25)}$. Mo- 
nitorar as alterações no perfil epidemiológico possibilita intensificar as ações de saúde a grupos mais acometidos e assim reduzir o impacto das complicações da doença.

Um percentual elevado de complicações agudas e a longo prazo do DM são observados nas internações, com consequente aumento do tempo de permanência e custo da hospitalização ${ }^{(2)}$. Na Finlândia, entre 1996 a 2011, a taxa de internação foi maior para diabetes sem complicações e nas complicações a longo prazo do diabetes ${ }^{(26)}$, similar ao encontrado no presente estudo.

Estudo $^{(6)}$ prévio demonstrou que a maioria das internações e readmissões foram por causas não relacionadas diretamente ao diabetes, sendo os idosos os mais acometidos, enquanto as pessoas na idade entre 18 a 44 anos estão mais propensos a serem readmitidos por graves alterações glicêmicas. No presente estudo, constatou-se uma importante redução nas complicações agudas, que estão associadas as alterações imediatas decorrentes do descontrole glicêmico.

Estudo $^{(23)}$ realizado em Lima, corrobora o nosso resultado quanto ao maior óbito durante a internação no sexo feminino e a complicações do diabetes a longo prazo. No entanto, esse estudo não constatou associação significativa entre o óbito e as variáveis sociodemográficas. O precário controle glicêmico é um fator que contribui para o aumento da taxa de mortalidade na internação, sendo observada alta letalidade da cetoacidose entre pessoas com idade acima de $75 \operatorname{anos}^{(7,22,27)}$.

Nossos resultados demonstram o maior peso da doença e da internação para os homens e pessoas com idade elevada, representando um maior risco de óbito durante a internação pela doença. Nos homens, as falhas no autocuidado e cuidado do controle glicêmico podem conduzir a complicações agudas da doença que estão associadas ao maior risco de óbito na internação. Para esses grupos em risco, diferentes estratégias podem ser usadas para possibilitar o cuidado continuado e o vínculo $^{(28,29)}$. O maior risco de óbito entre os idosos com DM pode ser decorrente das condições de fragilidade ${ }^{(30)}$, sendo necessário rastreio das fragilidades e cuidado adequado para reduzir o risco de hospitalização que pode contribuir para o óbito.

Na população do estudo, somente a raça/etnia não foi associada ao óbito. Os dados do estudo demonstram o grande número de registros sem esse dado, comprometendo dessa forma a qualidade da informação e as ações de saúde voltadas a grupos que pode estar em risco. Como demonstrado por estudo $^{(31)}$ prévio sobre desigualdades na taxa de internação entre grupos étnicos minoritários, procedentes de áreas mais carentes.

As desigualdades encontradas no padrão da taxa de internação entre as regiões de saúde do Pará corroboram os resultados encontrados entre as regiões de saúde do Paraná, em uma série temporal de 2000 a 2012. No início do período, a maior taxa foi de 26,9 internações por 10.000 habitantes e a menor foi de 5,4 internações por 10.000 habitantes, nas regiões de Umuarama e Foz do Iguaçu, respectivamente ${ }^{(18)}$.

No presente estudo as maiores taxas de internação e elevação durante o período do estudo foram observadas em locais com baixo acesso a ações e serviços de saúde, em todos os níveis de atenção, baixos indicadores sociais e de difícil acesso ${ }^{(13-14)}$. Há evidência na literatura sobre o impacto de bons indicadores sociais, como a escolaridade, na ocorrência de internação por $\mathrm{DM}^{(18,22)}$.

Essas desigualdades regionais podem ser reduzidas a partir de programa eficientes de desenvolvimento para a prevenção e tratamento de diabetes, como observado na Finlândia ${ }^{(26)}$. Assim como no Brasil, outros países também utilizam estratégias de rastreio e estratificação para que o tratamento seja adequado às necessidades de saúde de cada pessoa, no entanto, em alguns países a existência de diretrizes não foi capaz de reduzir a mortalidade associada ao $\mathrm{DM}^{(32)}$.

O estudo demostrou a evolução e magnitude das taxas de internação e mortalidade durante a internação no Pará; identificando grupos em riscos para o óbito durante a internação; e variação no padrão da taxa de internação nas regiões de saúde. Nesse contexto, para além do aumento da oferta de serviços de atenção à saúde em todos os níveis, torna-se relevante observar o modelo assistencial adotado, a estrutura física, processos de trabalho e a organização da linha de cuidado em cada região de saúde e macrorregiões.

Desta forma, somente a ampliação da oferta de serviço pode não ser garantia de acesso às ações de saúde de qualidade, principalmente, em um estado marcado por peculiaridades demográficas, epidemiológicas e da rede de serviços de saúde, que impactam diretamente nas condições de vida e saúde das populações ${ }^{(13-14)}$. Ademais, o monitoramento de indicadores positivos pode possibilitar o compartilhamento de experiências que reduzam o impacto dessa doença crônica para a pessoa e para o sistema de saúde.

O presente estudo teve como limitação a qualidade da informação coletada e introduzida no sistema de informação hospitalar, como a falta de disponibilidade de dados sobre a escolaridade e o baixo registro de dados sobre a raça/cor, que podem evidenciar as desigualdades entre os grupos populacionais, principalmente, quanto ao risco de óbito.

\section{CONCLUSÃO}

Nossos resultados demonstram um crescimento das taxas de internação e mortalidade durante a internação por DM até o ano de 2015. A redução seguinte pode ser reflexo dos processos 
de trabalho implantados a partir das mudanças que ocorreram nas diretrizes para o manejo da DM no Brasil. As internações foram mais frequentes em mulheres, pessoas com idade entre 60 a 69 anos, pardos e com complicações de longo prazo do DM. Contudo, o impacto da doença com maior chance de óbito na internação ocorreu entre os homens, idosos, além das complicações agudas e de longo prazo.

Nossos resultados demonstram disparidades na procedência das pessoas que internaram por DM no Pará. Estas desigualdades aumentaram ao longo do tempo em regiões de saúde com po- pulações mais vulneráveis. A detecção de áreas com taxas crescentes de internação é crucial para a implementação de intervenções específicas. Estratégias para reduzir a hospitalização por DM devem incluir a prevenção da doença, diagnóstico e tratamento precoces e prevenção de complicações.

\section{Referências}

1. Ministério da Saúde (BR). Portaria n. ${ }^{\circ} 221$, de 17 de abr. de 2008. Publica a Lista Brasileira de Internações por Condições Sensíveis à Atenção Primária. Brasília: Ministério da Saúde; 2008.

2. Bao X, Yang C, Fang K, Shi M, Yu G, Hu Y. Hospitalization costs and complications in hospitalized patients with type 2 diabetes mellitus in Beijing, China. J Diabetes 2016; 9(4):405-411.

3. Khalid JM, Raluy-Callado M, Curtis BH, Boye KS, Maguire A, Reaney M. Rates and risk of hospitalisation among patients with type 2 diabetes: retrospective cohort study using the UK General Practice Research Database linked to English Hospital Episode Statistics. Int J Clin Pract. 2013; 68(1):40-8.

4. Cavender MA, Steg PG, Smith SC Jr, Eagle K, Ohman EM, Goto S, Kuder J, Im K, Wilson PW, Bhatt DL. Impact of Diabetes Mellitus on Hospitalization for Heart Failure, Cardiovascular Events, and Death: Outcomes at 4 Years from the Reduction of Atherothrombosis for Continued Health (REACH) Registry. Circulation. 2015; 132(10):923-31.

5. Artilheiro MMVSA, Franco SC, Schulz VC, Coelho CC. Quem são e como são tratados os pacientes que internam por diabetes mellitus no SUS? Saúde Debate. 2014; 38(101), 210-224.

6. McCoy RG, Lipska KJ, Herrin J, Jeffery MM, Krumholz HM, Shah ND. Hospital Readmissions among Commercially Insured and Medicare Advantage Beneficiaries with Diabetes and the Impact of Severe Hypoglycemic and Hyperglycemic Events. J Gen Intern Med. 2017; 32(10):1097-1105.

7. Dolp R, Rehou S, Pinto R, Trister R, Jeschke MG. The effect of diabetes on burn patients: a retrospective cohort study. Crit Care. 2019; 23(1):28.

8. Santos FAL, Lima WP, Santos AL, Teston EF, Marcon SS. Hospitalizações por diabetes em adultos e idosos no Ceará, 2001-2012. Epidemiologia e Serviços de Saúde 2014; 23(4):655-663.

9. Santos AL, Teston EF, Latorre MRDO, Mathias THAF, Marcon SS. Tendência de hospitalizações por diabetes mellitus: implicações para o cuidado em saúde. Acta paul. Enferm 2015; 28(5):401-407.

10. Orozco-Beltrán $D$, Sánchez $E$, Garrido $A$, Quesada JA, Carratalá-Munuera MC, Gil-Guillén VF. Trends in Mortality from Diabetes Mellitus in Spain: 1998-2013. Revista Española de Cardiología 2017; 70(6):433-443, 2017.

11. Magalhães ALL, Morais Neto OL de. Desigualdades intraurbanas de taxas de internações por condições sensíveis à atenção primária na região central do Brasil. Ciênc. saúde coletiva. 2017; 22(6):2049-2062.

12. Mello JM, Borges PKO, Muller EV, Grden CRB, Pinheiro FK, Borges WS. Internações por Doenças Crônicas não Transmissíveis do Sistema Circulatório, Sensíveis a Atenção Primária a Saúde. Texto \& Contexto - Enfermagem. 2017; 26(1):e3390015.

13. Secretaria de Estado de Saúde Pública do Pará. Plano Estadual de Saúde do Pará 2016-2019. Belém: Secretaria de Estado de Saúde Pública do Pará, 2016.

14. Instituto Brasileiro de Geografia e Estatística. IBGE Cidades, 2019.

15. Prezotto KH, Chaves MMN, Mathias TAF. Hospitalizações sensiveis à atenção primária em crianças, segundo grupos etários e regionais de saúde. Revista da Escola de Enfermagem da USP. 2015; 49(1):44-53.

16. Sousa NP, Rehem TCMSB, Santos WS, Santos CE. Internações sensíveis à atenção primária à saúde em hospital regional do Distrito Federal. Re- vista Brasileira de Enfermagem. 2016; 69(1):118-125.

17. Costa JSD, et al. Tendência das internações por condição sensível à atenção primária e fatores associados em Porto Alegre, RS, Brasil. Ciência \& Saúde Coletiva. 2016; 21(4):1289-1296.

18. Arruda GO, Schmidt DB, Marcon SS. Internações por diabetes mellitus e a Estratégia Saúde da Família, Paraná, Brasil, 2000 a 2012. Ciência \& Saúde Coletiva. 2018; 23(2):543-552.

19. Santos BV dos, Lima DS, Fontes CJF. Internações por condições sensíveis à atenção primária no estado de Rondônia: estudo descritivo do período 2012-2016. Epidemiologia e Serviços de Saúde. 2019; 28(1):e2017497.

20. Ma J, Ward EM, Siegel RL, Jemal A. Temporal Trends in Mortality in the United States, 1969-2013. JAMA 2015; 27(16):1731-9.

21. Penso JM, Périco E. Mortalidade por diabetes mellitus em uma macrorregião de saúde de Minas Gerais. Revista de Enfermagem e atenção à saúde. 2016; 5(2):34-44.

22. Chen PC, Tsai CY, Woung LC, Lee YC. Socioeconomic disparities in preventable hospitalization among adults with diabetes in Taiwan: a multilevel modelling approach. Int J Equity Health. 2015; 14:31.

23. Zelada H, Bernabe-Ortiz A, Manrique H. In hospital Mortality in Patients with Type 2 Diabetes Mellitus: A Prospective Cohort Study in Lima, Peru. J Diabetes Res. 2015; 2016:7287215.

24. Gerhardt PC, Borghi AC, Fernandes CAM, Mathias TAF, Carreira L. Tendência das internações por diabetes mellitus e hipertensão arterial sistêmica em idosos. Cogitare Enfermagem. 2016; 21(4):01-10.

25. Comino EJ, Harris MF, Islam MD, Tran DT, Jalaludin B, Jorm L, Flack J, Haas M. Impact of diabetes on hospital admission and length of stay among a general population aged 45 year or more: a record linkage study. BMC Health Serv Res. 2015; 15:12.

26. Manderbacka K, Arffman M, Lumme S, Lehikoinen M, Winell K, Keskimäki I. Regional trends in avoidable hospitalisations due to complications among population with diabetes in Finland in 1996-2011: a register-based cohort study. BMJ Open. 2016;6(8):e011620.

27. Benoit SR, Zhang Y, Geiss LS, Gregg EW, Albright A. Trends in Diabetic Ketoacidosis Hospitalizations and In-Hospital Mortality - United States, 2000-2014. MMWR Morb Mortal Wkly Rep. 2018; 67:362-365.

28. Arruda GO, Marcon SS. Inquérito sobre a utilização dos serviços de saúde por homens adultos: prevalências e fatores associados. Rev. Latino-Am. Enfermagem. 2016; 24:e2685.

29. Moreira MMCN, Gomes R, Ribeiro CR. E agora o homem vem?! Estratégias de atenção à saúde dos homens. Cadernos de Saúde Pública. 2016; 32(4):e00060015.

30. Antunes JFS, Okuno MFP, Lopes MCBT, Campanhar CRV, Batista REA. Frailty Assessment of Elderly Hospitalized at an Emergency Service of a University Hospital. Cogitare Enferm. 2015; 20(2):264-71.

31. Nishino Y, Gilmour S, Shibuya K. Inequality in diabetes-related hospital admissions in England by socioeconomic deprivation and ethnicity: facility-based cross-sectional analysis. PLoS One. 2015; 10(2):e0116689. 32. Selph S, Dana T, Blazina I, Bougatsos C, Patel H, Chou R. Screening for type 2 diabetes mellitus: a systematic review for the U.S. Preventive Services Task Force. Ann Intern Med. 2015 Jun 2; 162(11):765-76. 